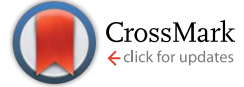

Cite this: RSC Adv., 2017, 7, 16890

Received 28th January 2017

Accepted 9th March 2017

DOI: 10.1039/c7ra01222e

rsc.li/rsc-advances

\section{Enhanced photoresponse of $\mathrm{ZnO}$ quantum dot- decorated $\mathrm{MoS}_{2}$ thin films $\uparrow$}

\author{
Ghazanfar Nazir, ${ }^{a}$ M. Farooq Khan, ${ }^{a}$ Imtisal Akhtar, ${ }^{\mathrm{b}}$ Kamran Akbar, ${ }^{\text {a }}$ Praveen Gautam, ${ }^{\text {a }}$ \\ Hwayong Noh, ${ }^{a}$ Yongho Seo, ${ }^{b}$ Seung-Hyun Chun ${ }^{a}$ and Jonghwa Eom ${ }^{\star a}$
}

\begin{abstract}
Transition metal dichalcogenides (TMDs) have been attracting attention because of their applications in optoelectronics and photo-detection. A widely used TMD semiconductor is molybdenum disulfide $\left(\mathrm{MoS}_{2}\right)$, which has tremendous applications because of its tunable bandgap and high luminescence quantum efficiency. This paper reports on high photo responsivity $\left(R_{\lambda} \sim 1913 \mathrm{~A} \mathrm{~W}^{-1}\right)$ of $\mathrm{MoS}_{2}$ photodetector by decorating a thin layer of zinc oxide $(\mathrm{ZnO})$ quantum dots ( $\mathrm{ZnO}-\mathrm{QDs}$ ) on $\mathrm{MoS}_{2}$. Results show that $R_{\lambda}$ increases dramatically to $2267 \mathrm{~A} \mathrm{~W}^{-1}$ at $V_{\mathrm{bg}}=30 \mathrm{~V}$. The high response of ZnO-QDs/MoS heterostructures is attributed to a number of factors, such as effective charge transfer between $\mathrm{ZnO}$ QDs and $\mathrm{MOS}_{2}$ surface and re-absorption of light photon resulting in production of electron-hole pairs.
\end{abstract}

\section{Introduction}

Photovoltaic cells, photodetectors, sensors, light-emitting diodes (LEDs), and LED displays will eventually require optoelectronic materials that demonstrate more efficient characteristics than those currently used. Graphene is an emergent material, which has been widely studied ${ }^{1-3}$ because of its promising properties, such as high mobility, ultra-thinness, and flexibility. ${ }^{4,5}$ Graphene has been extensively used in photodetection, ${ }^{6}$ bioelectronics, ${ }^{7}$ optoelectronics, ${ }^{8}$ and gas sensing. ${ }^{9}$

Transition metal dichalcogenide (TMD) thin films have been used in the development of nano- and opto-electronic devices, such as ambipolar and high-quality field-effect transistors, ${ }^{\mathbf{1 0}, 11}$ digital integrated circuits, ${ }^{12}$ electric double-layer transistors, ${ }^{13}$ and highly responsive ${ }^{14}$ photodetectors. ${ }^{15}$ TMD materials have been widely investigated through theoretical or experimental studies, such as the investigation on charge transfer and photon-exciton interactions. ${ }^{\mathbf{1 6 - 2 1}}$ One of the basic methods to tune the optical properties of TMDs involves controlling the charge carrier density. One of the mostly used TMD materials that has been widely investigated is molybdenum disulfide $\left(\mathrm{MoS}_{2}\right)$. Different methods used to inject charge carriers in TMDs were intensively studied; these methods include tuning of charge carriers by using back gate voltage, ${ }^{22} \mathrm{O}_{2}$ and $\mathrm{H}_{2} \mathrm{O}$ molecule adsorption, ${ }^{23,24}$ chemical process of molecule interaction, ${ }^{25}$ and plasmonic hot electron doping. ${ }^{26}$ Thus, finding

${ }^{a}$ Department of Physics \& Astronomy, Graphene Research Institute, Sejong University, Seoul 05006, Korea. E-mail: eom@sejong.ac.kr

${ }^{b}$ Department of Nanotechnology \& Advanced Materials Engineering, Graphene Research Institute, Sejong University, Seoul 05006, Korea

$\dagger$ Electronic supplementary information (ESI) available. See DOI: 10.1039/c7ra01222e a suitable means of efficient doping to achieve superior optical properties of TMDs is necessary.

Semiconductor quantum dots (QDs) demonstrate unique behaviors, such as size-tunable atomic-like characteristics resulting from quantum confinement in the nanometer scale. A number of semiconductors consisting of QDs have been widely used in different research fields because of their anomalous behavior. Groups II-VI semiconductor QDs, such as CdSe and $\mathrm{ZnSe}^{27,28}$ displays an advantage over IV and III-V materials ${ }^{29-31}$ due to their higher exciton energies and the stronger phononexciton interaction among them. Unfortunately, the bandgap of CdSe in bulk form is $1.74 \mathrm{eV}$, which is very difficult to tune in the ultraviolet (UV) region. Moreover, CdSe is toxic and unsafe for medical applications. On contrary, ZnSe can be used in UV-blue range of energies. However, ZnSe-based devices demonstrate less efficient performance because of certain defects. Zinc oxide (ZnO), which displays a wide direct bandgap of $3.37 \mathrm{eV}$ at room temperature, is a good candidate for short wavelength applications. $\mathrm{ZnO}$ has become famous as the brightest emitter among available wide-bandgap semiconductors because of its high exciton energy $(\sim 60 \mathrm{meV}) .{ }^{32}$ In addition, $\mathrm{ZnO}$ is low cost and exhibits high resistance to defects, high stability, environment friendly characteristics, and biosafety. Based on these characteristics, ZnO-QDs are advantageous over CdSe-QDs and ZnSe-QDs in terms of practical applications. ${ }^{33,34}$

This study comparatively explored the photoresponse of pristine $\mathrm{MoS}_{2}$ and $\mathrm{ZnO}-\mathrm{QDs} / \mathrm{MoS}_{2}$ heterostructures. We investigated a number of electrical and photoelectrical properties, such as carrier mobility, responsivity $\left(R_{\lambda}\right)$, detectivity $\left(D^{*}\right)$, external quantum efficiency, and linear dynamic range with and without ZnO-QDs. All of these parameters were investigated at various back gate voltages $\left(V_{\mathrm{bg}}\right)$. High carrier mobility when $\mathrm{ZnO}-\mathrm{QDs}$ is deposited over $\mathrm{MoS}_{2}$ is caused by reduced carrier 
transit time (the time required for an electron or other charge carrier to travel between two electrodes in a transistor) within a field-effect transistor (FET). Moreover, rise and decay times of carriers in a photodetector was calculated, and the results showed that after ZnO-QDs interacted with pristine $\mathrm{MoS}_{2}$, the decay time dramatically changed suggesting the efficient charge transfer that occurred between $\mathrm{ZnO}$-QDs and $\mathrm{MoS}_{2}$ surface. Furthermore, this work discusses the proposed mechanism of charge transfer between $\mathrm{ZnO}-\mathrm{QDS}$ and pristine $\mathrm{MoS}_{2}$.

\section{Experimental section}

\section{Sample preparation}

Naturally available $\mathrm{MoS}_{2}$ was mechanically exfoliated using the scotch tape method over a heavily p-doped Si as substrate with $300 \mathrm{~nm}$-thick $\mathrm{SiO}_{2}$ as capping layer. The desired flake with a suitable thickness was chosen by using an optical microscope. A large $\mathrm{Cr} / \mathrm{Au}$ pattern with a thickness of $6 / 30 \mathrm{~nm}$ was subsequently deposited around the desired flake by using photolithography. To make source and drain contacts, we performed ebeam lithography, followed by the final deposition of an 8/80 nm-thick $\mathrm{Cr} / \mathrm{Au}$ in an evaporation chamber, in which a high vacuum of $2 \times 10^{-6}$ Torr was maintained. Electrical measurements using Keithley 2400 Source Meter and Keithley 6485K Picoammeter were subsequently performed by placing the sample in a vacuum at room temperature. To study photoresponse, we placed our sample in a vacuum and illuminated by deep UV light (DUV) with an intensity of $11 \mathrm{~mW} \mathrm{~cm}^{-2}$ and a wavelength of $220 \mathrm{~nm}$. Structural investigation and material identification were performed using Raman spectroscopy and atomic force microscopy (AFM). Fig. S1† shows the results of the Raman analysis of our flake, and the results confirm the multilayer nature of $\mathrm{MoS}_{2}$ with peaks located in exactly the same positions as described in a number of publications. Laser wavelength was obtained at $514 \mathrm{~nm}$, and a low power of less than $1 \mathrm{~mW}$ was chosen to avoid structural degradation caused by heating effects of laser. The size of laser spot used in Raman spectroscopy is $0.7 \mu \mathrm{m}$. Fig. S2 $\uparrow$ shows the AFM micrograph of $\mathrm{MoS}_{2}$, and the image confirms the multilayer nature of $\mathrm{MoS}_{2}$ which is $7 \mathrm{~nm}$ thick ( 11 layers).

\section{Synthesis and characterization of ZnO QDs}

ZnO-QDs were synthesized in methanol via hydrolysis method. Typically, $20 \mathrm{mmol}$ methanol solution of $\mathrm{ZnAc}_{2}: 2 \mathrm{H}_{2} \mathrm{O}$ was prepared and maintained at $60^{\circ} \mathrm{C}$ followed by dropwise addition of $200 \mathrm{mmol}$ methanol solution of $\mathrm{KOH}$ for $10 \mathrm{~min}$ under vigorous stirring for $2 \mathrm{~h}$. Finally, the QDs were collected by centrifugation and then washed multiple times with methanol. The ZnO-QDs solution was drop casted over $\mathrm{MoS}_{2}$ photodetector device and then baked gently at $70{ }^{\circ} \mathrm{C}$ for $10 \mathrm{~min}$. To confirm the nature of the material, we measured the excitation and emission of matrix (EEM) of ZnO-QDs by using a spectrofluorometer (Hitachi, F7000, Japan). The measured excitation/emission wavelength was adjusted to $220-500 \mathrm{~nm} / 280-550 \mathrm{~nm}$ at a scan step of 5 and $1 \mathrm{~nm}$ for excitation and emission, respectively. Moreover, a polarized cut off filter of $290 \mathrm{~nm}$ was placed in front of a lamp to remove Rayleigh scattering, and Milli-Q water was used as blank and subtracted from the EEM of each sample.

\section{Results and discussion}

Fig. 1a shows the 3D schematic of a photodetector consisting of ZnO-QDs decorated $\mathrm{MoS}_{2}$ as channel flake. The $\mathrm{MoS}_{2}$ is supported on $\mathrm{SiO}_{2} / \mathrm{p}^{+}$-Si wafer substrate with 300 nm-thick $\mathrm{SiO}_{2}$. Fig. 1b shows the optical image of the $\mathrm{MoS}_{2}$ FET with a channel length of $1.52 \mu \mathrm{m}$ and a width of $6.34 \mu \mathrm{m}$.

Raman spectroscopy is the most useful tool utilized in nondestructive analysis of structural properties..$^{35-42}$ We chose a desired flake by using an optical microscope and then performed Raman analysis to confirm flake thickness and to identify the material. The Raman spectra of the $\mathrm{MoS}_{2}$ flake were obtained at room temperature. Fig. S1 $\uparrow$ shows the Raman spectra for our $\mathrm{MoS}_{2}$ flake. The in-plane $\left(\mathrm{E}_{2 \mathrm{~g}}^{1}\right)$ and out-of-plane $\left(\mathrm{A}_{1 \mathrm{~g}}\right)$ vibrational modes for multilayer $\mathrm{MoS}_{2}\left(\mathrm{ML}-\mathrm{MoS}_{2}\right)$ was found at $\sim 384$ and $\sim 408 \mathrm{~cm}^{-1}$, respectively. The difference between $\mathrm{E}_{2 \mathrm{~g}}^{1}$ and $\mathrm{A}_{1 \mathrm{~g}}$ of the $\mathrm{ML}-\mathrm{MoS}_{2}$ is $\sim 24 \mathrm{~cm}^{-1}$, consistent with previous results. ${ }^{43-45}$ The black curve represents pristine $\mathrm{MoS}_{2}$ and the red curve represents the $\mathrm{ZnO}-\mathrm{QDs} / \mathrm{MoS}_{2}$ heterostructures. The peak positions did not change after $\mathrm{ZnO}$ deposition. Fig. S2 $\uparrow$ shows the AFM images used to further confirm the thickness of the layer of the ML-MoS ${ }_{2}$ flake. The measured thickness of our flake is $7 \mathrm{~nm}$ (11-layer). We found that the average height of $\mathrm{ZnO}-\mathrm{QDs}$ is $2-4 \AA$ and the average width is 10$20 \mathrm{~nm}$ (Fig. S3†).

We determined the electrical characteristics under vacuum by applying $V_{\mathrm{bg}}$. Transfer characteristics of pristine $\mathrm{MoS}_{2}$ and $\mathrm{ZnO}-\mathrm{QDS} / \mathrm{MoS}_{2}$ structures were examined and the results are shown in Fig. 1c. We maintained the drain-source voltage $\left(V_{\mathrm{ds}}\right)$ at $0.5 \mathrm{~V}$ throughout our measurements. The drain current $\left(I_{\mathrm{ds}}\right)$ increases after drop casting $\mathrm{ZnO}-\mathrm{QDS}$ over $\mathrm{MoS}_{2}$ in positive gate voltage range while decrease in negative gate voltage because of increase in leakage current in that region. The field-effect mobilities can be calculated using the fundamental formula $\mu=\frac{L}{W}\left(\frac{\mathrm{d} I_{\mathrm{ds}}}{\mathrm{d} V_{\mathrm{bg}}}\right) \frac{1}{V_{\mathrm{ds}} C_{\mathrm{g}}}$, where $L$ and $W$ are the channel length $(1.52 \mu \mathrm{m})$ and width $(6.34 \mu \mathrm{m})$ of our $\mathrm{MoS}_{2}$ flake, respectively, $\frac{\mathrm{d} I_{\mathrm{ds}}}{\mathrm{d} V_{\mathrm{bg}}}$ is the slope of the linear region of transfer curves, and $C_{\mathrm{g}}$ is

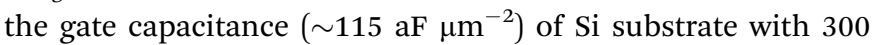
nm-thick $\mathrm{SiO}_{2}$ as capping layer. Therefore, the field-effect mobilities are 5.75 and $25.09 \mathrm{~cm}^{2} \mathrm{~V}^{-1} \mathrm{~s}^{-1}$ before and after decorating ZnO-QDs on $\mathrm{MoS}_{2}$ flake, respectively.

Fig. $2 \mathrm{a}$ and $\mathrm{b}$ show the output $(I-V)$ characteristics before and after drop casting ZnO-QDs over $\mathrm{MoS}_{2}$ surface. Output characteristics of pristine $\mathrm{MoS}_{2}$ show a linear relationship with $I_{\mathrm{ds}}$ and $V_{\mathrm{ds}}$ at different $V_{\mathrm{bg}}$ values, demonstrating that contacts $(\mathrm{Cr} / \mathrm{Au})$ on the surface of $\mathrm{MoS}_{2}$ are ohmic and that no Schottky barrier exists at the metal-semiconductor junction interface. Moreover, Fig. 2b shows the output characteristics after decorating ZnO-QDs over $\mathrm{MoS}_{2}$. The output characteristics again show ohmic nature of the contacts but with increased $I_{\mathrm{ds}}$, confirming that ZnO-QDs on the surface of $\mathrm{MoS}_{2}$ 
(a)

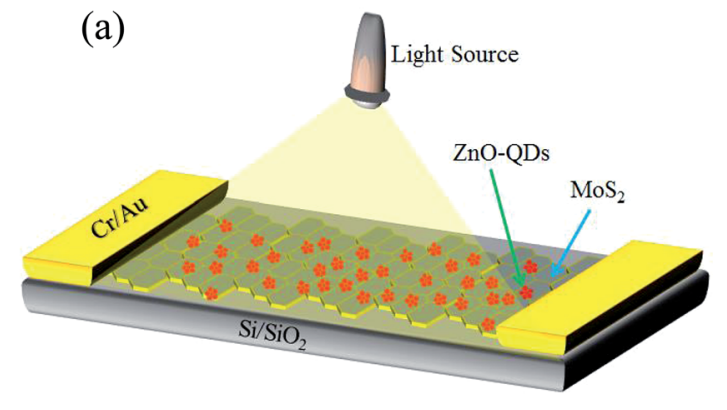

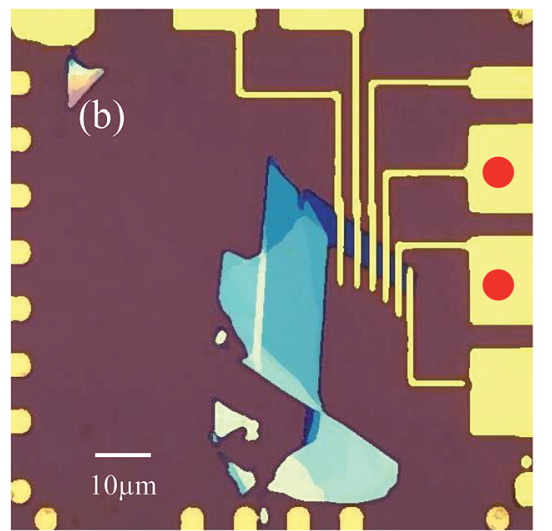

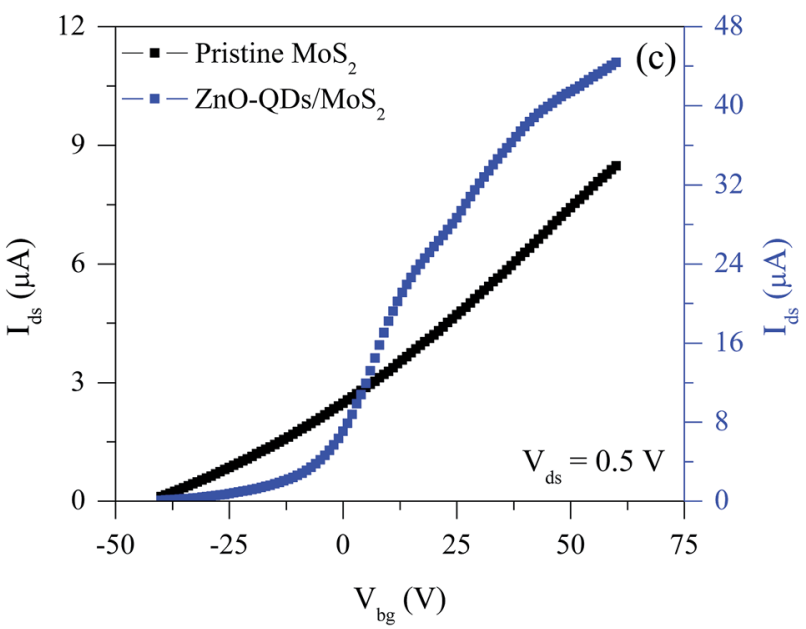

Fig. 1 (a) 3D schematic of $\mathrm{ZnO}-\mathrm{QDs}$ decorated $\mathrm{MoS}_{2}$ photodetector and (b) optical image of $\mathrm{MoS}_{2}$ (scale bar: $10 \mu \mathrm{m}$ ) consisting of a multilayer flake with $\mathrm{Cr} / \mathrm{Au}=8 / 60 \mathrm{~nm}$ contacts. Red circles represent the contacts for electrical measurements. (c) $I_{\mathrm{ds}}$ versus $V_{\mathrm{bg}} \mathrm{graph}$ for MoS $\mathrm{S}_{2}$ photodetector measured at $V_{\mathrm{ds}}=0.5 \mathrm{~V}$ without and with $\mathrm{ZnO}-\mathrm{QDs}$. The mobility of pristine $\mathrm{MoS}_{2}$ and $\mathrm{ZnO}-\mathrm{QDs} / \mathrm{MoS}_{2}$ heterostructures are 5.75 and $25.09 \mathrm{~cm}^{2} \mathrm{~V}^{-1} \mathrm{~s}^{-1}$, respectively.
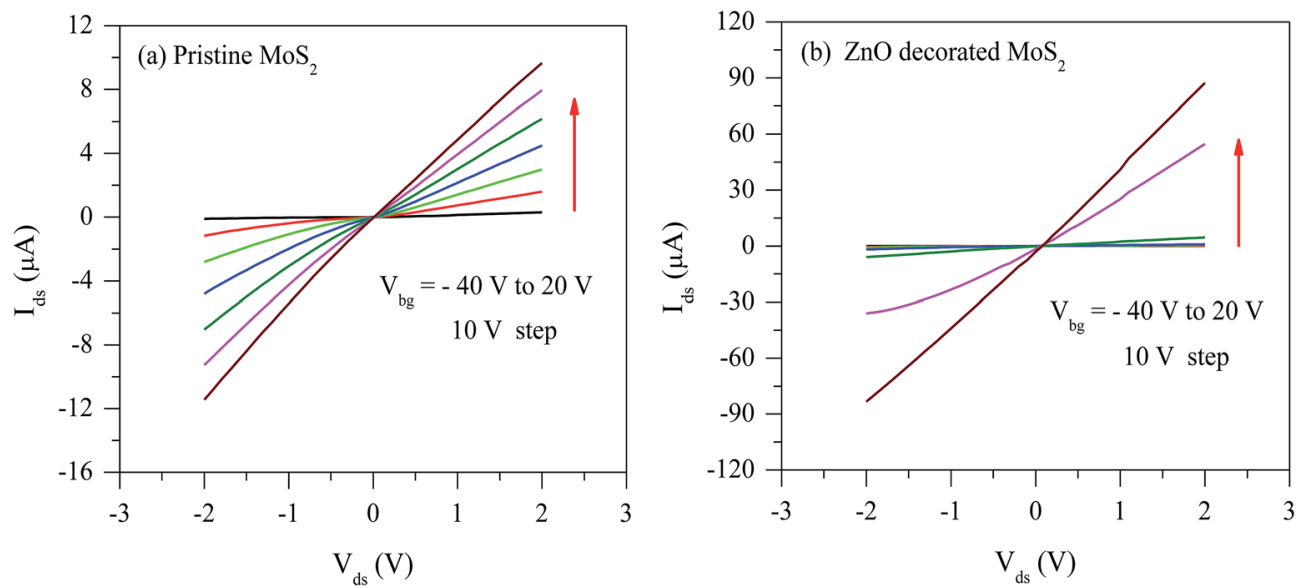

Fig. 2 (a) Output characteristics $\left(I_{\mathrm{ds}}-V_{\mathrm{ds}}\right)$ of pristine $\mathrm{MoS}_{2}$ at different $V_{\mathrm{bg}}$ values with equal steps of $10 \mathrm{~V}$. (b) The corresponding $\left(/_{\mathrm{ds}}-V_{\mathrm{ds}}\right)$ curves after $\mathrm{ZnO}-\mathrm{QDs}$ decoration at the same $V_{\mathrm{bg}}$ showed an increase in current. The graphs in (a) and (b) show a linear trend, confirming the formation of ohmic contacts before and after decorating $\mathrm{ZnO}-\mathrm{QDs}$ on $\mathrm{MoS}_{2}$.

does not damage the contacts or cause structural deformation, which is beneficial for the applications of FET in optoelectronics.
We further investigated $\mathrm{MoS}_{2}$ photodetectors by determining the time-dependent photoresponse of pristine $\mathrm{MoS}_{2}$ and $\mathrm{ZnO}-\mathrm{QDs} / \mathrm{MoS}_{2}$ heterostructures under varying $V_{\mathrm{bg}}$ values. To 
determine the photoresponse behavior of our device, we placed our device in a vacuum and illuminated by DUV in a way that light falls vertically on its surface with an effective area of 9.64 $\mu \mathrm{m}^{2}$. All measurements were performed at $V_{\mathrm{ds}}=0.5 \mathrm{~V}$. As DUV light falls on $\mathrm{MoS}_{2}$ surface, the photocurrent $\left(I_{\mathrm{Ph}}\right)$ increases with increasing $V_{\text {bg }}$ caused by the increase in carrier drift velocity and the corresponding reduction in carrier transit time (defined as $T_{\mathrm{t}}=L^{2} / \mu V_{\mathrm{ds}}{ }^{14}$ where $\mu$ is the field-effect mobility and $L$ is the channel length). Our calculation showed that the carrier transit time for $\mathrm{ZnO}-\mathrm{QDS} / \mathrm{MoS}_{2}$ is lower than that for pristine $\mathrm{MoS}_{2}$. As a result, the current increases with increasing $V_{\mathrm{bg}}$; thus, $I_{\mathrm{Ph}}$ becomes dominant over thermionic and tunneling currents at all $V_{\mathrm{bg}}$ values. ${ }^{46}$

$I_{\mathrm{Ph}}$ for pristine $\mathrm{MoS}_{2}$ and $\mathrm{ZnO}-\mathrm{QDs} / \mathrm{MoS}_{2}$ are shown in Fig. 3a and b, respectively, and $I_{\mathrm{Ph}}$ of $\mathrm{ZnO}-\mathrm{QDS} / \mathrm{MoS}_{2}$ is greater than that of pristine $\mathrm{MoS}_{2}$ at all $V_{\mathrm{bg}}$ values $(0-30 \mathrm{~V})$. This phenomenon was observed because in the case of $\mathrm{ZnO}-\mathrm{QDS} / \mathrm{MoS}_{2}$ heterostructures, ZnO-QDs provide surplus carriers to $\mathrm{MoS}_{2}$, and these carriers equally contribute to the increase in overall photoresponse behavior under DUV light. ${ }^{47}$ Another important parameter in evaluating devices under light illumination is $R_{\lambda}$. $R_{\lambda}$ indicates the response of a device to light (specific wavelength) and is defined as " $I_{\mathrm{Ph}}$ produced per unit power of incident light on effective area of a photodetector". ${ }^{46}$

$$
R_{\lambda}=\frac{\Delta I_{\mathrm{Ph}}}{P A}
$$

where $P$ is the light intensity, $A$ is the effective area of photodetector, and $\Delta I_{\mathrm{Ph}}$ is the photocurrent generation $\left(\Delta I_{\mathrm{Ph}}=I_{\mathrm{Ph}}-\right.$ $\left.I_{\text {dark }}\right) . R_{\lambda}$ is highly dependent on wavelength of incident light. Responsivity $\left(R_{\lambda}\right)$ is negligible for a light of wavelengths $>680 \mathrm{~nm},{ }^{14}$ which corresponds to an energy level of $1.8 \mathrm{eV}$, the bandgap for monolayer $\mathrm{MoS}_{2}$. Thus, to excite the electrons from the valence band to the conduction band, we must use a light of shorter wavelength. $R_{\lambda}$ of our photodetector with an effective area of $9.46 \mu \mathrm{m}^{2}$ was measured under DUV light with an intensity $11 \mathrm{~mW} \mathrm{~cm}^{-2}$ and a wavelength of $220 \mathrm{~nm} . \Delta I_{\mathrm{Ph}}$ and the corresponding $R_{\lambda}$ of pristine $\mathrm{MoS}_{2}$ and $\mathrm{ZnO}-\mathrm{QDS} / \mathrm{MoS}_{2}$ are shown in Fig. $4 \mathrm{a}$ and b respectively. $\Delta I_{\mathrm{Ph}}$ and $R_{\lambda}$ both increased in $\mathrm{ZnO}-\mathrm{QDS} / \mathrm{MoS}_{2}$ heterostructures at all $V_{\mathrm{bg}}$ values because of the increase in charge carriers transferred from ZnO-QDs to $\mathrm{MoS}_{2}$ surface (see Table S1 in ESI $\dagger$ ). We measured the dark current without and with $\mathrm{ZnO}-\mathrm{QDs}$, and Fig. 4c shows that the dark current is higher after decorating ZnO-QDs over $\mathrm{MoS}_{2}$ at all $V_{\text {bg }}$ values. We also measured detectivity $\left(D^{*}\right)$ as shown in Fig. 4 d, which is defined as the "ability of a device to detect weak optical signal". Given that dark current mainly contributes to noise factor of photodetector, then $D^{*}$ is expressed as follows: ${ }^{48}$

$$
D^{*}=\frac{R_{\lambda} A^{1 / 2}}{\sqrt{2 e I_{\mathrm{dark}}}}
$$

where $R_{\lambda}$ is responsivity, $e$ is the electronic charge, and $I_{\text {dark }}$ is the current in the absence of light. $D^{*}$ is expressed in jones, where 1 jones $=1 \mathrm{~cm} \mathrm{~Hz}^{1 / 2} \mathrm{~W}^{-1}$. In this study, $D^{*}$ is in the order of $10^{11}$ jones. Low $D^{*}$ of heterostructures at low $V_{\mathrm{bg}}$ values (Fig. 4d) is due to the increase in dark current at these voltages (Fig. 4c).

We prepared a schematic of energy band (Fig. 5) to illustrate photodetector behavior of our pristine $\mathrm{MoS}_{2}$. In the absence of light illumination and any $V_{\mathrm{ds}}$ or $V_{\mathrm{bg}}$, the device is at equilibrium state. When light with a wavelength of $220 \mathrm{~nm}$ falls on pristine $\mathrm{MoS}_{2}$ in its OFF state $\left(V_{\mathrm{bg}}=0 \mathrm{~V}\right)$, light is absorbed by the pristine $\mathrm{MoS}_{2}$, resulting in the excitation of electrons to the conduction band even when $V_{\text {bg }}$ was zero (Fig. 5b). A low number of charge carriers will move to the conduction band when $V_{\mathrm{bg}}<V_{\text {th }}$ and the drain current is very low (Fig. 5c). In contrast, in the ON state $\left(V_{\mathrm{bg}}>V_{\mathrm{th}}\right)$, photo-generated, thermionic, and tunneling currents contribute cumulatively to $I_{\mathrm{Ph}}$ enhancement (Fig. 5d). Our devices showed good $R_{\lambda}$ at room temperature relative to that of the reported devices. ${ }^{\mathbf{4 9 , 5 0}}$

Dynamic response to light irradiation was studied, and the rise and decay times were measured by fitting the curves to exponential decay function ${ }^{51,52}$
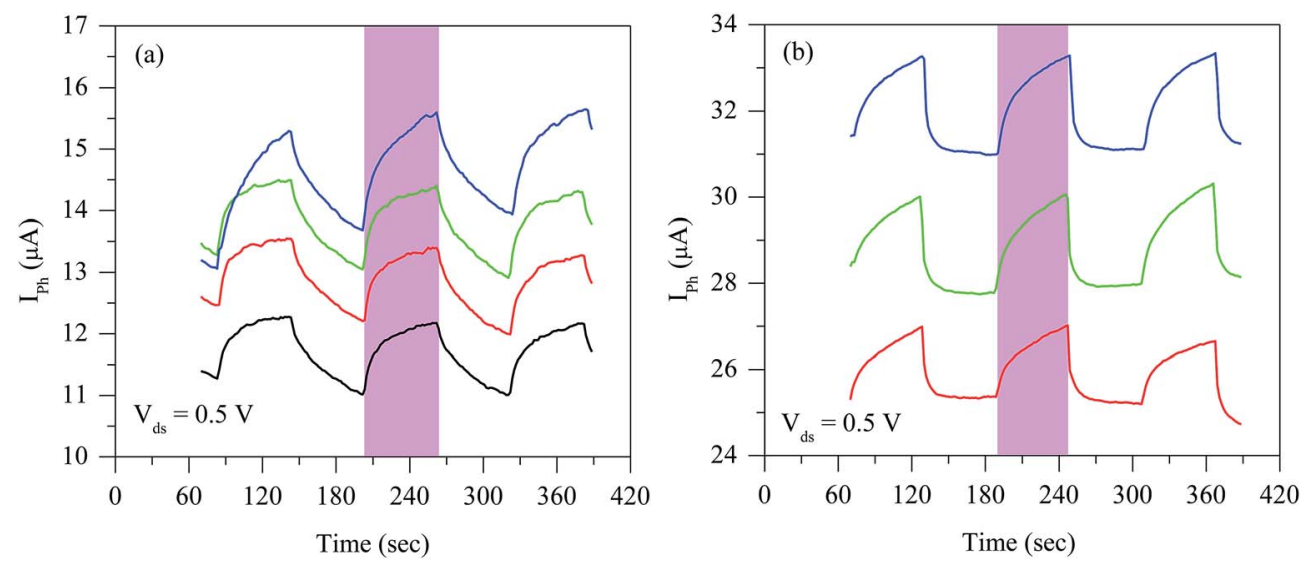

Fig. 3 Photocurrent $\left(I_{\mathrm{Ph}}\right)$ versus time under different $V_{\mathrm{bg}}$ values ranging from $V_{\mathrm{bg}}=0 \mathrm{~V}$ (black curve) to $V_{\mathrm{bg}}=30 \mathrm{~V}$ (blue curve). Photoresponse of (a) pristine $\mathrm{MoS}_{2}$ measured at different $V_{\mathrm{bg}}$ values with equal steps of $10 \mathrm{~V}$ and $V_{\mathrm{ds}}=0.5 \mathrm{~V}$ under deep ultraviolet illumination with an intensity of 11 $\mathrm{mW} \mathrm{cm}{ }^{-2}$ and a wavelength of $220 \mathrm{~nm}$ with alternating on and off cycles. (b) The photoresponse of the same device after ZnO-QDs drop casting shows higher $I_{\mathrm{Ph}}$ at all $V_{\mathrm{bg}}$ values. The photoresponse at $V_{\mathrm{bg}}=0 \mathrm{~V}$ has been omitted in order to see the clear interpretation of plot. 

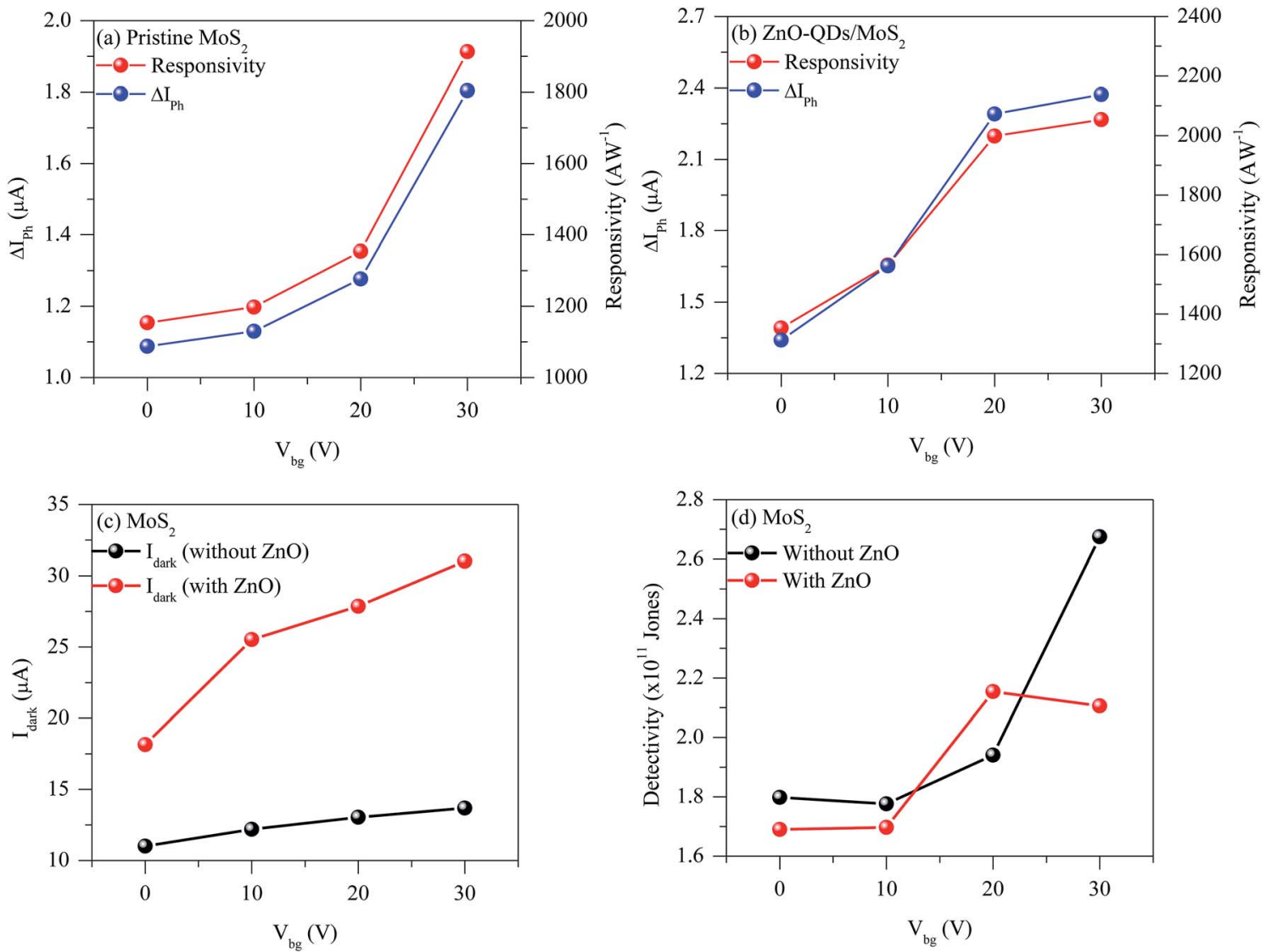

Fig. 4 Photocurrent generation $\left(\Delta /_{\mathrm{Ph}}\right)$ (in blue circles) and responsivity $\left(R_{\lambda}\right)$ (in red circles) versus back gate voltage $\left(V_{\mathrm{bg}}\right)$ for (a) pristine MoS $S_{2}$ and (b) $\mathrm{ZnO}-\mathrm{QDs} / \mathrm{MoS}_{2}$ heterostructures; $\Delta /_{\mathrm{Ph}}$ and $R_{\lambda}$ both increased after decorating ZnO-QD on $\mathrm{MoS}_{2}$. (c) Dark current versus $V_{\mathrm{bg}}$ before and after ZnO-QD decoration. Dark current is higher in ZnO-QDs/MoS $S_{2}$ heterostructure than in pristine $\mathrm{MoS}_{2}$ at all $V_{\mathrm{bg}}$ values. (d) Detectivity ( $D^{*}$ ) versus $V_{\mathrm{bg}}$ before and after $\mathrm{ZnO}-\mathrm{QDs}$ at a power of $10^{11}$ jones. Low value of $D^{*}$ at a low $V_{\mathrm{bg}}$ is caused by high dark current at these values.

$$
I_{\mathrm{Ph}}(t)=I_{\text {dark }}+A \exp \left(\frac{-t}{\tau_{\text {decay }}}\right)
$$

where $A$ is the scaling constant, $\tau_{\text {decay }}$ is the time constant for decay, and $t$ is the time after switching on or off of DUV light. We can determine the time constant $(\tau)$ by fitting the experimental curves. The decay and rise times at different $V_{\mathrm{bg}}$ values showed similar average values, indicating that $V_{\mathrm{bg}}$ does not considerably contribute to the changes in decay and rise times. Fig. 6 shows the rise and decay times at different $V_{\mathrm{bg}}$ values before and after decorating with ZnO-QDs. For pristine $\mathrm{MoS}_{2}$, the rise time at each $V_{\mathrm{bg}}$ is lower than the corresponding decay times (Fig. 6a and c). Our results are consistent with previous findings. ${ }^{14}$ In $I_{\mathrm{Ph}}$ dynamics, environmental effects are very important in a photodetector. The decay time can be varied in the range of $0.3-4000 \mathrm{~s}$ under various surface treatments probably because of the difference in surface hydrophobicity. ${ }^{53,54}$ The device response of our pristine $\mathrm{MoS}_{2}$ is slow, with rise and decay times longer than $\sim 12$ and $\sim 26 \mathrm{~s}$ at $V_{\mathrm{bg}}=0 \mathrm{~V}$, respectively. This slow response is attributed to either charge impurity states or defects in the bandgap or to the existence of trap states ${ }^{14,43,55}$ between the underlying $\mathrm{SiO}_{2}$ layer and $\mathrm{MoS}_{2}$. To further enhance the $R_{\lambda}$ and degrade the response time, we spread ZnO-QDs over $\mathrm{MoS}_{2}$ surface to form heterostructures.
The considerable change in transfer characteristics of $\mathrm{ZnO}$ $\mathrm{QDs} / \mathrm{MoS}_{2}$ heterostructures is convincing. Fig. $6 \mathrm{~b}$ and $\mathrm{d}$ show the rise and decay times for $\mathrm{ZnO}-\mathrm{QDs} / \mathrm{MoS}_{2}$ heterostructures. The rise time $(\sim 24 \mathrm{~s})$ of our heterostructures at $V_{\mathrm{bg}}=0 \mathrm{~V}$ increases to more than $\sim 12 \mathrm{~s}$ (for pristine $\mathrm{MoS}_{2}$ ) because of the surplus charge carriers transferred from ZnO-QDs to $\mathrm{MoS}_{2}$ surface. Moreover, drain current significantly increases in ZnO$\mathrm{QDs} / \mathrm{MoS}_{2}$ heterostructures possibly caused by the increase in carrier concentration resulting from the transfer of charge carriers from ZnO-QDs to pristine $\mathrm{MoS}_{2}$. Another notable phenomenon is the reduction in decay time in $\mathrm{ZnO}-\mathrm{QDs} / \mathrm{MoS}_{2}$ heterostructures; fast decay is speculated to be related to direct recombination of light-excited carriers and to sub-bandgap emissions caused by the existence of charge impurity and trap states in the bandgap of $\mathrm{MoS}_{2}$ surface. ${ }^{56}$ This fast decay is related to the efficient charge transfer between $\mathrm{MoS}_{2}$ surface and ZnO-QDs. Oxygen molecules adsorb onto oxide surfaces and thus occupy free electrons available in n-type metal oxide semiconductors, along with the formation of less conductive depletion layer near ZnO-QD surface. In ZnO-QDs, existence of hole trap states is highly probable because of high surface-tovolume ratio, ${ }^{57}$ consistent with the 3D-PL spectrum shown in Fig. S4. $\dagger$ When these heterostructures are illuminated under a light with a photon energy that is larger than the ZnO 
(a)

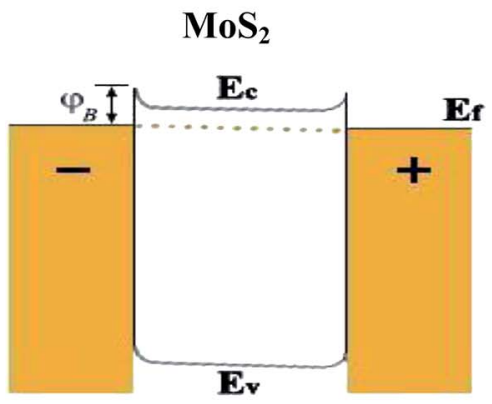

\section{Without Illumination Open Circuit Under Equilibrium}

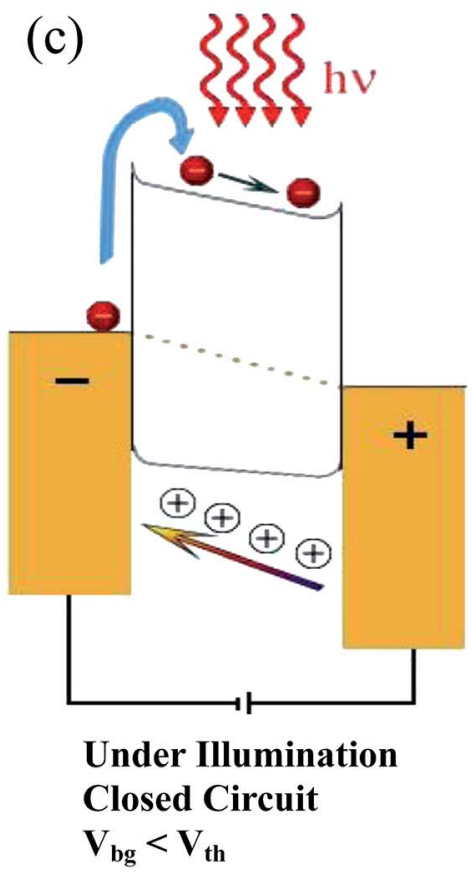

(b)

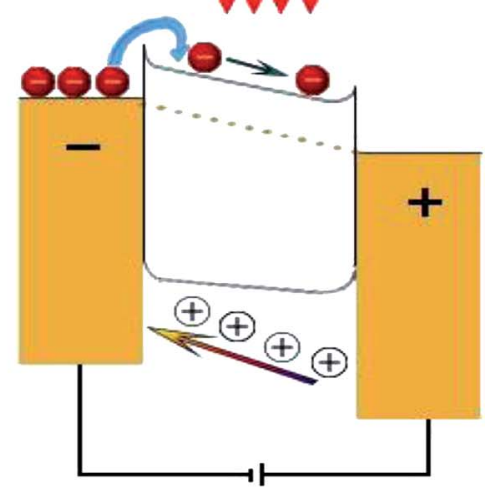

Under Illumination

Closed Circuit

$\mathrm{V}_{\text {bg }}=\mathbf{0 ~ V}$

(d)

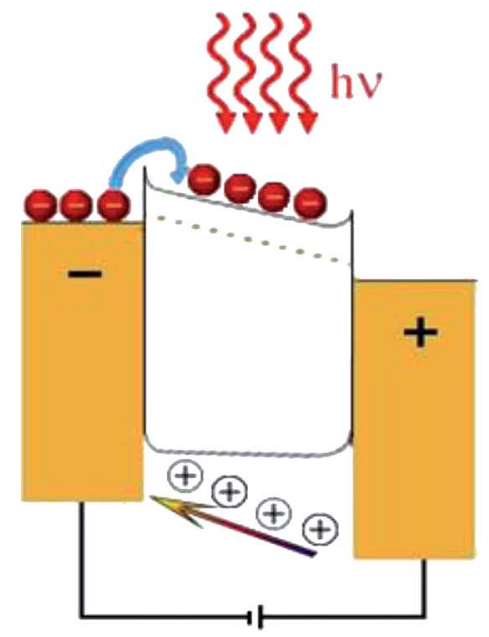

Under Illumination

Closed Circuit

$\mathbf{V}_{\text {bg }}>\mathbf{V}_{\text {th }}$

Fig. 5 Schematic energy diagram in the absence of light and under illumination represent the energy barrier and mechanism of photocurrent $\left(I_{\mathrm{Ph}}\right)$ generation. Schematic energy diagram (a) in the dark and at drain-source voltage $\left(V_{\mathrm{ds}}\right)=0 \mathrm{~V}$ under equilibrium and (b) under light illumination at $V_{\mathrm{ds}} \neq 0 \mathrm{~V}$ and back gate voltage $\left(V_{\mathrm{bg}}\right)=0$. (c and d) $I_{\mathrm{Ph}}$ generation under light illumination at different $V_{\mathrm{bg}}$ values for $V_{\mathrm{bg}}\left\langle V_{\mathrm{th}}\right.$ and $V_{\mathrm{bg}}>$ $V_{\text {th }}$ respectively by taking $V_{\mathrm{ds}} \neq 0 \mathrm{~V}$.

bandgap, electron-hole pairs are produced and immediately separated from the holes trapped at the surface (caused by band bending) leaving behind unpaired electrons that quickly transfer to $\mathrm{MoS}_{2}$ channel and are gathered by means of $V_{\mathrm{ds}}$. The trapped holes recombine with negatively charged oxygen ions, producing neutral oxygen molecules, which are then desorbed from the surface of ZnO-QDs. Water and oxygen molecules present in air also physisorb at $\mathrm{MoS}_{2} / \mathrm{SiO}_{2}$ interface and create p-doping $\mathrm{MoS}_{2}$ channel. ${ }^{47}$ However, deposition of ZnO-QDs onto $\mathrm{MoS}_{2}$ surface causes Dirac point to shift from high voltage to low voltage because of electron transfer from $\mathrm{ZnO}$ QDs to $\mathrm{MoS}_{2}$ surface, compensating the doping of physisorbed oxygen at the $\mathrm{ZnO}-\mathrm{QDS} / \mathrm{MoS}_{2}$ channel surface.

To understand the mechanism of $I_{\mathrm{Ph}}$ generation in our heterostructures, we prepared a schematic of energy band (Fig. 7). Fig. 7 shows the band bending and carrier transfer direction when $\mathrm{ZnO}$-QDs and $\mathrm{MoS}_{2}$ interact with each other. The electron affinity ${ }^{56}$ of $\mathrm{ML} \mathrm{MoS}_{2}$ is approximately $3.9 \mathrm{eV}$, which is comparable to that of ZnO-QDs. Our $\mathrm{ML} \mathrm{MoS}_{2}$ film is $7 \mathrm{~nm}$ thick ( $\sim 11$ layers) and its indirect bandgap ${ }^{56}$ is $1.2 \mathrm{eV}$, which is 

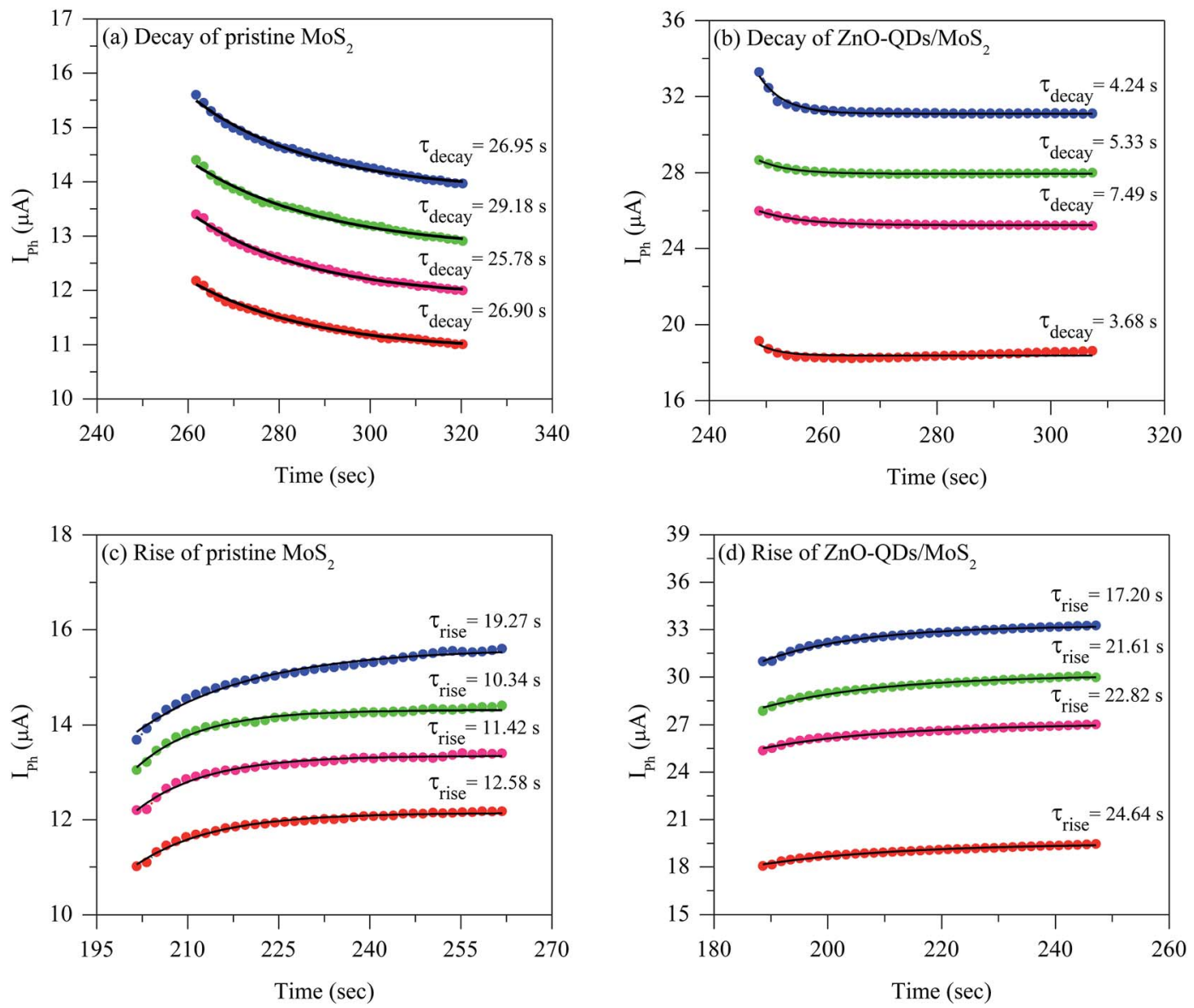

Fig. 6 Photocurrent $\left(I_{\mathrm{Ph}}\right)$ versus time at different back gate voltages $\left(V_{\mathrm{bg}}\right)$ ranging from $V_{\mathrm{bg}}=0 \mathrm{~V}$ (red curve) to $V_{\mathrm{bg}}=30 \mathrm{~V}$ (blue curve). (a) $I_{\mathrm{Ph}}$ relaxation for pristine $\mathrm{MoS}_{2}$ at different $V_{\mathrm{bg}}$ values in the absence of DUV light. (b) Dramatic increase in $/ \mathrm{ph}$ relaxation for $\mathrm{ZnO}-\mathrm{QDs} / \mathrm{MoS}$ at all $V_{\mathrm{bg}}$ values. (c) Rise time for pristine $\mathrm{MoS}_{2}$ under DUV light at different $V_{\mathrm{bg}}$ values. (d) Rise time after ZnO-QDs decoration over MoS ${ }_{2}$. The data were fitted by eqn (3) and indicated by black lines in all graphs.

(a) Before Contact

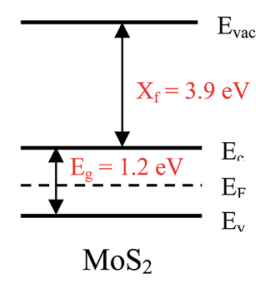

$\mathrm{MoS}_{2}$

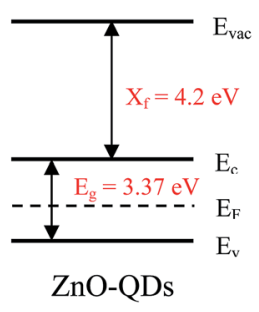

ZnO-QDs (b) Formation of $\mathrm{MoS}_{2} / \mathrm{ZnO}-\mathrm{QDs}$ Heterojunction

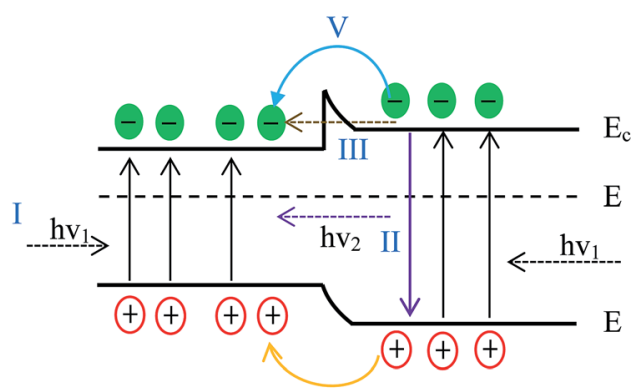

$\mathrm{MoS}_{2}$
IV

$\mathrm{ZnO}-\mathrm{QDs}$

Fig. 7 (a) Energy diagram for $\mathrm{MoS}_{2}$ and $\mathrm{ZnO}$ quantum dots (QDs) before contact. (b) Energy diagram of the interface between $\mathrm{MoS}_{2}$ and $\mathrm{ZnO}$ QDs after construction of heterojunction; different Roman numerals represent different proposed mechanisms of carrier transformation across junction, as well as between valence and conduction bands. (I) Shows photon excitation of charge carriers in $\mathrm{MoS}_{2}$ and $\mathrm{ZnO}$-QDs; (II) indicates that carriers de-excite the photon emitted by $\mathrm{ZnO}-\mathrm{QDs}$ and that the emitted photon is re-absorbed by $\mathrm{MoS}_{2}$; (III) electrons tunnel from $\mathrm{ZnO}$ QDs to $\mathrm{MoS}_{2}$; (IV) holes transfer from ZnO-QDs to $\mathrm{MoS}_{2}$; and (V) electrons from ZnO-QDs are thermally agitated toward $\mathrm{MoS}_{2}$.

considerably smaller than the bandgap of ZnO-QDs $(3.37 \mathrm{eV}) .^{58}$ Considering that both $\mathrm{ZnO}-\mathrm{QDs}$ and $\mathrm{MoS}_{2}$ are n-type materials, we proposed energy band diagrams showing the bandgap before and after formation of heterostructures (Fig. 7a and b).
Fermi level for ZnO-QDs is closer to vacuum level as compared with that for $\mathrm{MoS}_{2}$ (Fig. 7). Fig. 7a shows basic schematic for $\mathrm{MoS}_{2}$ and ZnO-QDs contains information about electron affinity and bandgap before their heterojunction. When $\mathrm{MoS}_{2}$ and $\mathrm{ZnO}$ - 
QDs interact to form heterostructures caused by van der Waals forces, we proposed that there exist number of ways of carrier injection between $\mathrm{MoS}_{2}$ and ZnO-QDs. When light falls on heterostructure, there occurs injection of electrons from $\mathrm{ZnO}$ QDs conduction band into $\mathrm{MoS}_{2}$ conduction band as explained by process " $V$ " in Fig. $7 \mathrm{~b}$ and leads to increase in current. Also, some of the electrons in ZnO-QDs conduction band get energy from thermal agitation at room temperature and move to $\mathrm{MoS}_{2}$ conduction band as explained by process "III". The electrons in the valence band also move from $\mathrm{ZnO}$ QDs to $\mathrm{MoS}_{2}$ due to thermal agitation shown by process "IV". The motion of electrons causes band bending, ${ }^{59}$ which is manifested as a small energy barrier. When these heterostructures are illuminated under DUV light, photo-generation occurs because both ZnO-QDs and $\mathrm{MoS}_{2}$ strongly absorb light photon and electrons move from valence to conduction band explained by process "I". Also, some of the electrons in the conduction band move back to the valence band of ZnO-QDs and emit light photon which caught by electrons in the valence band of $\mathrm{MoS}_{2}$ and get excited toward conduction band of $\mathrm{MoS}_{2}$ explained by process "II". $I_{\mathrm{Ph}}$ enhancement is generally caused by a large number electron-hole pairs, resulted from tunneling of electrons from ZnO-QDs to $\mathrm{MoS}_{2}$ surface. Given that the decay time of the heterostructures is shorter than that of pristine $\mathrm{MoS}_{2}$, the recombination rate and consequently the photoresponse is faster in $\mathrm{ZnO}-\mathrm{QDs} / \mathrm{MoS}_{2}$ than in pristine $\mathrm{MoS}_{2}$.

Two more parameters, namely, external quantum efficiency (EQE) and linear dynamic response (LDR) were investigated to better assess the photodetector performance. These parameters were measured at different $V_{\mathrm{bg}}$ values without and with interaction of ZnO-QDs. EQE is given by the formula ${ }^{48}$

$$
\mathrm{EQE}=\frac{h c R_{\lambda}}{e \lambda}
$$

EQE is defined as the "number of photo-induced carriers per incident photons" $h$ is Planck's constant, $c$ is the speed of light, $e$ is the elementary charge, $R_{\lambda}$ is the photo responsivity, and $\lambda$ is the wavelength of illumination. Fig. 8a shows the EQE values versus $V_{\mathrm{bg}}$ with and without $\mathrm{ZnO}$-QDs. EQE values at all $V_{\mathrm{bg}}$ values are higher in $\mathrm{ZnO}-\mathrm{QDs} / \mathrm{MoS}_{2}$ than in pristine $\mathrm{MoS}_{2}$. EQE can be improved by increasing $R_{\lambda}$ factor and by irradiating the samples with incident light with short wavelength. The high EQE values for $\mathrm{ZnO}-\mathrm{QDS} / \mathrm{MoS}_{2}$ heterostructures are mainly caused by the increase in photo-induced charge carriers, as well as by accumulation of carriers by $\mathrm{ZnO}-\mathrm{QDs}$. Another factor that we investigated is $\mathrm{LDR}^{\mathbf{4 8 , 6 0}}$ which is the maximum linear response of a detector relative to its noise. LDR or photosensitive linearity measures the image quality in biomedical image processing and sensing and is given by the following formula:

$$
\mathrm{LDR}=20 \log \left(\frac{I_{\mathrm{Ph}}}{I_{\mathrm{dark}}}\right)
$$

where $I_{\mathrm{Ph}}$ is the value of current measured by our device under illumination with a light intensity of $11 \mathrm{~mW} \mathrm{~cm}^{-2}$ and $I_{\text {dark }}$ is the dark current at different $V_{\mathrm{bg}}$ values in the absence of light. Fig. 8b compares LDR versus $V_{\mathrm{bg}}$ of the pristine $\mathrm{MoS}_{2}$ and $\mathrm{ZnO}-$ QDs/ $\mathrm{MoS}_{2}$ heterostructures. The LDR values of the heterostructures are low because of low $\left(\frac{I_{\mathrm{Ph}}}{I_{\mathrm{dark}}}\right)$ ratios. In other words, the increase in dark current in $\mathrm{ZnO}-\mathrm{QDs} / \mathrm{MoS}_{2}$ heterostructures (Fig. 4c) degrades LDR.

To confirm the size of ZnO-QDs, we obtained an AFM image (Fig. S3 $\dagger$ ), which shows that the average height of ZnO-QDs is 2-4 $\AA$ and the average width is $10-20 \mathrm{~nm}$. Moreover, we obtained a 3D fluorescence plot by choosing a range of wavelength of light to be used to irradiate our samples (Fig. S4 $\dagger$ ). By using the formula $E_{\mathrm{g}}=h c / \lambda$, we determined the height excitation peak at $\sim 378 \mathrm{~nm}$, leading to a bandgap of $3.28 \mathrm{eV}$, which corresponds to the bandgap of our $10 \mathrm{~nm}$ ZnO-QDs. ${ }^{61}$ The EEM of ZnO-QDs was measured using a spectrofluorometer. The EEM clearly shows the maximum emission peak at approximately $440 \mathrm{~nm}$ at $378 \mathrm{~nm}$ excitation. The red thick line is caused by Raman scattering of ZnO-QDs dispersed in methanol solution.
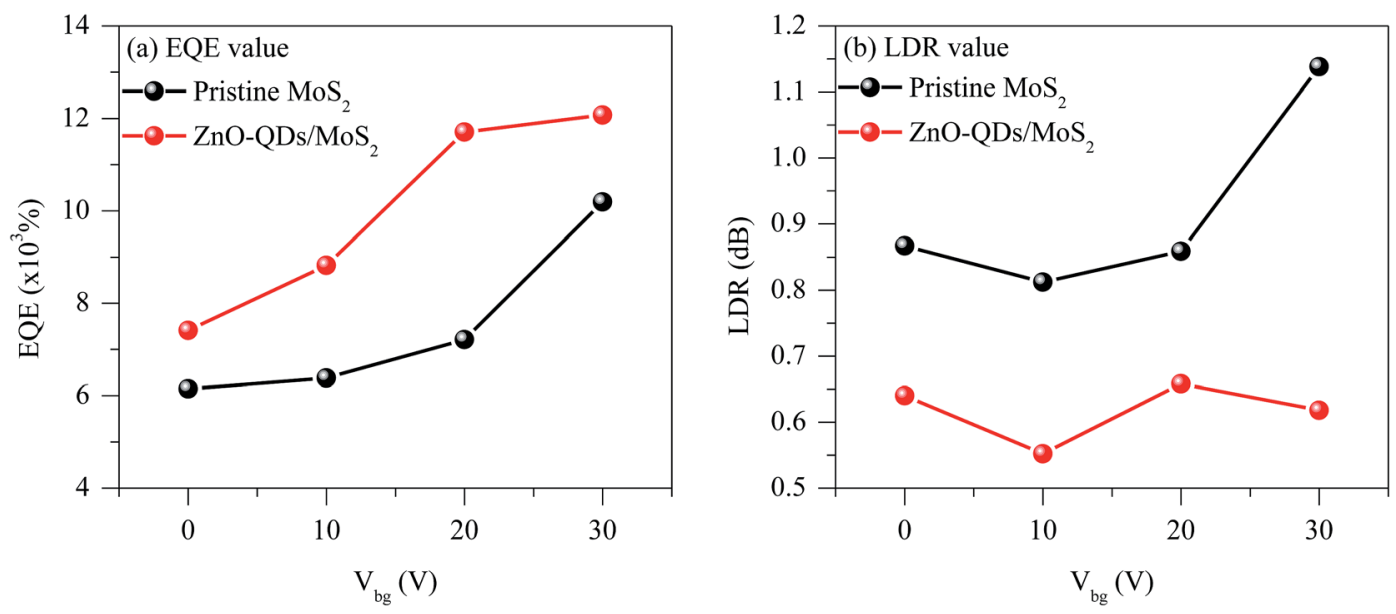

Fig. 8 (a) External quantum efficiency (EQE) versus back gate voltage $\left(V_{b g}\right)$ for pristine-MoS ${ }_{2}$ and $Z n O-Q D s / M o S_{2}$. EQE is high after $Z n O$ decoration over $\mathrm{MoS}_{2}$. (b) Linear dynamic range (LDR) versus $V_{\mathrm{bg}}$ for pristine $\mathrm{MoS}_{2}$ and $\mathrm{ZnO}-\mathrm{QDs} / \mathrm{MoS}_{2}$. LDR decreases after ZnO-QD decoration at all $V_{\mathrm{bg}}$ values because of the increase in dark current as shown in Fig. 4c. 


\section{Conclusion}

We investigated for the first time a high-response photodetector based on $\mathrm{MoS}_{2} / \mathrm{ZnO}-\mathrm{QDS}$ heterostructures. The junction between $\mathrm{MoS}_{2}$ and $\mathrm{ZnO}-\mathrm{QDs}$ forms $\mathrm{n}-\mathrm{n}$ type heterostructures, resulting in enhanced carrier mobility caused by injection of electrons from ZnO-QDs into $\mathrm{MoS}_{2}$. A number of factors were investigated. $R_{\lambda}$ increased after ZnO-QDs were deposited over $\mathrm{MoS}_{2}$ surface, and this phenomenon resulted from the increase in $I_{\mathrm{Ph}}$ caused by the surplus carriers cumulatively transferred from ZnO-QDs to $\mathrm{MoS}_{2}$ surface. EQE increased after ZnO-QDs were drop casted over $\mathrm{MoS}_{2}$, whereas after decorating with ZnO-QDs, $D^{*}$ decreased caused by the massive increase in dark current. Moreover, LDR degradation corresponds to the increase in dark current in $\mathrm{ZnO}-\mathrm{QDS} / \mathrm{MoS}_{2}$ heterostructures. We extensively discussed the basic phenomenon of charge transfer in pristine $\mathrm{MoS}_{2}$ and $\mathrm{ZnO}-\mathrm{QDs} / \mathrm{MoS}_{2}$ heterostructures. In addition, this report presents a number of proposed factors that contribute to the increasing $I_{\mathrm{Ph}}$ resulting from generation of charge carriers across the junction of the ZnO-QDs and $\mathrm{MoS}_{2}$ surfaces. We suggest that generation of charge carriers, which contribute to $I_{\mathrm{Ph}}$ enhancement, is affected by the five parameters mentioned above (Fig. 7). This high photoresponse is largely caused by the effect of light-matter interactions based on tunneling of photo-excited carriers from $\mathrm{ZnO}-\mathrm{QDs}$ to $\mathrm{MoS}_{2}$ and by re-absorption of emitted photons from ZnO-QDs by $\mathrm{MoS}_{2}$. Our work describes the basic mechanism of charge transfer between ZnO-QDs and $\mathrm{MoS}_{2}$.

\section{Acknowledgements}

This research was supported by Nano-Material Technology Development Program (2012M3A7B4049888) through the National Research Foundation of Korea (NRF) funded by the Ministry of Science, ICT and Future Planning. This research was also supported by Priority Research Center Program (20100020207) and the Basic Science Research Program (2016R1D1A1A09917762) through NRF funded by the Ministry of Education.

\section{References}

1 N. Mohanty and V. Berry, Graphene-based single-bacterium resolution biodevice and DNA transistor: interfacing graphene derivatives with nanoscale and microscale biocomponents, Nano Lett., 2008, 8(12), 4469-4476.

2 S. Rumyantsev, G. Liu, M. S. Shur, R. A. Potyrailo and A. A. Balandin, Selective gas sensing with a single pristine graphene transistor, Nano Lett., 2012, 12(5), 2294-2298.

3 C.-H. Liu, Y.-C. Chang, T. B. Norris and Z. Zhong, Graphene photodetectors with ultra-broadband and high responsivity at room temperature, Nat. Nanotechnol., 2014, 9(4), 273-278.

4 F. Schwierz, Graphene transistors, Nat. Nanotechnol., 2010, 5(7), 487-496.

5 A. K. Geim and K. S. Novoselov, The rise of graphene, Nat. Mater., 2007, 6(3), 183-191.
6 J. Park, Y. Ahn and C. Ruiz-Vargas, Imaging of photocurrent generation and collection in single-layer graphene, Nano Lett., 2009, 9(5), 1742-1746.

7 L. H. Hess, M. Seifert and J. A. Garrido, Graphene transistors for bioelectronics, Proc. IEEE, 2013, 101(7), 1780-1792.

8 F. Bonaccorso, Z. Sun, T. Hasan and A. Ferrari, Graphene photonics and optoelectronics, Nat. Photonics, 2010, 4(9), 611-622.

9 Y. Liu, J. Chang and L. Lin, in A flexible graphene FET gas sensor using polymer as gate dielectrics, 2014 IEEE 27th International Conference on Micro Electro Mechanical Systems (MEMS), IEEE, 2014, pp. 230-233.

10 V. Podzorov, M. Gershenson, C. Kloc, R. Zeis and E. Bucher, High-mobility field-effect transistors based on transition metal dichalcogenides, Appl. Phys. Lett., 2004, 84(17), 33013303.

11 B. Radisavljevic and A. Kis, Mobility engineering and a metal-insulator transition in monolayer $\mathrm{MoS}_{2}$, Nat. Mater., 2013, 12(9), 815-820.

12 H. Wang, L. Yu, Y.-H. Lee, Y. Shi, A. Hsu, M. L. Chin, L.-J. Li, M. Dubey, J. Kong and T. Palacios, Integrated circuits based on bilayer $\mathrm{MoS}_{2}$ transistors, Nano Lett., 2012, 12(9), 46744680 .

13 Y. Zhang, J. Ye, Y. Yomogida, T. Takenobu and Y. Iwasa, Formation of a stable $\mathrm{p}-\mathrm{n}$ junction in a liquid-gated $\mathrm{MoS}_{2}$ ambipolar transistor, Nano Lett., 2013, 13(7), 3023-3028.

14 O. Lopez-Sanchez, D. Lembke, M. Kayci, A. Radenovic and A. Kis, Ultrasensitive photodetectors based on monolayer $\mathrm{MoS}_{2}$, Nat. Nanotechnol., 2013, 8(7), 497-501.

15 Z. Yin, H. Li, H. Li, L. Jiang, Y. Shi, Y. Sun, G. Lu, Q. Zhang, $\mathrm{X}$. Chen and H. Zhang, Single-layer $\mathrm{MoS}_{2}$ phototransistors, ACS Nano, 2011, 6(1), 74-80.

16 Q. H. Wang, K. Kalantar-Zadeh, A. Kis, J. N. Coleman and M. S. Strano, Electronics and optoelectronics of twodimensional transition metal dichalcogenides, Nat. Nanotechnol., 2012, 7(11), 699-712.

17 S. Z. Butler, S. M. Hollen, L. Cao, Y. Cui, J. A. Gupta, H. R. Gutierrez, T. F. Heinz, S. S. Hong, J. Huang and A. F. Ismach, Progress, challenges, and opportunities in two-dimensional materials beyond graphene, ACS Nano, 2013, 7(4), 2898-2926.

18 Z. J. Qi, J. A. Rodríguez-Manzo, A. s. R. Botello-Méndez, S. J. Hong, E. A. Stach, Y. W. Park, J.-C. Charlier, M. Drndić and A. C. Johnson, Correlating atomic structure and transport in suspended graphene nanoribbons, Nano Lett., 2014, 14(8), 4238-4244.

19 C. Cong, J. Shang, X. Wu, B. Cao, N. Peimyoo, C. Qiu, L. Sun and T. Yu, Synthesis and Optical Properties of Large-Area Single-Crystalline 2D Semiconductor WS2 Monolayer from Chemical Vapor Deposition, Adv. Opt. Mater., 2014, 2(2), 131-136.

20 A. Rubio, Hybridized graphene: nanoscale patchworks, Nat. Mater., 2010, 9(5), 379-380.

21 Q. Tang and Z. Zhou, Graphene-analogous low-dimensional materials, Prog. Mater. Sci., 2013, 58(8), 1244-1315.

22 J. S. Ross, S. Wu, H. Yu, N. J. Ghimire, A. M. Jones, G. Aivazian, J. Yan, D. G. Mandrus, D. Xiao and W. Yao, 
Electrical control of neutral and charged excitons in a monolayer semiconductor, Nat. Commun., 2013, 4, 1474.

23 S. Tongay, J. Zhou, C. Ataca, J. Liu, J. S. Kang, T. S. Matthews, L. You, J. Li, J. C. Grossman and J. Wu, Broad-range modulation of light emission in two-dimensional semiconductors by molecular physisorption gating, Nano Lett., 2013, 13(6), 2831-2836.

24 H. Nan, Z. Wang, W. Wang, Z. Liang, Y. Lu, Q. Chen, D. He, P. Tan, F. Miao and X. Wang, Strong photoluminescence enhancement of $\mathrm{MoS}_{2}$ through defect engineering and oxygen bonding, ACS Nano, 2014, 8(6), 5738-5745.

25 S. Mouri, Y. Miyauchi and K. Matsuda, Tunable photoluminescence of monolayer $\mathrm{MoS}_{2}$ via chemical doping, Nano Lett., 2013, 13(12), 5944-5948.

26 Y. Kang, S. Najmaei, Z. Liu, Y. Bao, Y. Wang, X. Zhu, N. J. Halas, P. Nordlander, P. M. Ajayan and J. Lou, Plasmonic hot electron induced structural phase transition in a $\mathrm{MoS}_{2}$ monolayer, Adv. Mater., 2014, 26(37), 6467-6471.

27 I. Suemune, T. Tawara, T. Saitoh and K. Uesugi, Stability of CdSe and ZnSe dots self-organized on semiconductor surfaces, Appl. Phys. Lett., 1997, 71(26), 3886-3888.

28 N. Myung, Y. Bae and A. J. Bard, Effect of surface passivation on the electrogenerated chemiluminescence of CdSe/ZnSe nanocrystals, Nano Lett., 2003, 3(8), 1053-1055.

29 A. Malachias, R. Magalhães-Paniago, S. Kycia and D. G. Cahill, X-ray study of strain and composition of $\mathrm{Si}$ / Ge0. 85Si0. 15(111) islands grown in Volmer-Weber mode, J. Appl. Phys., 2004, 96(6), 3234-3238.

30 A. Tsatsul'nikov, A. Kovsh, A. Zhukov, Y. M. Shernyakov, Y. G. Musikhin, V. Ustinov, N. Bert, P. Kop'ev, Z. I. Alferov and A. Mintairov, Volmer-Weber and Stranski-Krastanov InAs-(Al, Ga) As quantum dots emitting at $1.3 \mu \mathrm{m}, \mathrm{J}$. Appl. Phys., 2000, 88(11), 6272-6275.

31 S. Fafard, D. Leonard, J. Merz and P. Petroff, Selective excitation of the photoluminescence and the energy levels of ultrasmall InGaAs/GaAs quantum dots, Appl. Phys. Lett., 1994, 65(11), 1388-1390.

32 Z. Tang, G. K. Wong, P. Yu, M. Kawasaki, A. Ohtomo, H. Koinuma and Y. Segawa, Room-temperature ultraviolet laser emission from self-assembled $\mathrm{ZnO}$ microcrystallite thin films, Appl. Phys. Lett., 1998, 72(25), 3270-3272.

33 Z. L. Wang, Zinc oxide nanostructures: growth, properties and applications, J. Phys.: Condens. Matter, 2004, 16(25), R829.

34 J. Lu, Z. Ye, Y. Zhang, Q. Liang, S. Fujita and Z. Wang, Selfassembled ZnO quantum dots with tunable optical properties, Appl. Phys. Lett., 2006, 89(2), 023122.

35 D. J. Late, Temperature dependent phonon shifts in singlelayer WS2, ACS Appl. Mater. Interfaces, 2014, 6(2), 1158-1163.

36 D. J. Late, S. N. Shirodkar, U. V. Waghmare, V. P. Dravid and C. Rao, Thermal Expansion, Anharmonicity and TemperatureDependent Raman Spectra of Single-and Few-Layer MoSe2 and WSe2, ChemPhysChem, 2014, 15(8), 1592-1598.

37 J. K. Ellis, M. J. Lucero and G. E. Scuseria, The indirect to direct band gap transition in multilayered $\mathrm{MoS}_{2}$ as predicted by screened hybrid density functional theory, Appl. Phys. Lett., 2011, 99(26), 261908.
38 A. Kuc, N. Zibouche and T. Heine, Influence of quantum confinement on the electronic structure of the transition metal sulfide T S 2, Phys. Rev. B: Condens. Matter Mater. Phys., 2011, 83(24), 245213.

39 A. Castellanos-Gomez, R. Roldán, E. Cappelluti, M. Buscema, F. Guinea, H. S. van der Zant and G. A. Steele, Local strain engineering in atomically thin $\mathrm{MoS}_{2}$, Nano Lett., 2013, 13(11), 5361-5366.

40 R. Yan, J. R. Simpson, S. Bertolazzi, J. Brivio, M. Watson, X. Wu, A. Kis, T. Luo, A. R. H. Walker and H. G. Xing, Thermal conductivity of monolayer molybdenum disulfide obtained from temperature-dependent Raman spectroscopy, ACS Nano, 2014, 8(1), 986-993.

41 Y. Du, H. Liu, A. T. Neal, M. Si and D. Y. Peide, Molecular doping of multilayer field-effect transistors: reduction in sheet and contact resistances, IEEE Electron Device Lett., 2013, 34(10), 1328-1330.

42 M. Thripuranthaka, R. V. Kashid, C. S. Rout and D. J. Late, Temperature dependent Raman spectroscopy of chemically derived few layer $\mathrm{MoS}_{2}$ and WS2 nanosheets, Appl. Phys. Lett., 2014, 104(8), 081911.

43 W. Zhang, J. K. Huang, C. H. Chen, Y. H. Chang, Y. J. Cheng and L. J. Li, High-Gain Phototransistors Based on a CVD $\mathrm{MoS}_{2}$ Monolayer, Adv. Mater., 2013, 25(25), 3456-3461.

44 C. Lee, H. Yan, L. E. Brus, T. F. Heinz, J. Hone and S. Ryu, Anomalous lattice vibrations of single-and few-layer $\mathrm{MoS}_{2}$, ACS Nano, 2010, 4(5), 2695-2700.

45 H. Li, Q. Zhang, C. C. R. Yap, B. K. Tay, T. H. T. Edwin, A. Olivier and D. Baillargeat, From bulk to monolayer $\mathrm{MoS}_{2}$ : evolution of Raman scattering, Adv. Funct. Mater., 2012, 22(7), 1385-1390.

46 M. A. Shehzad, S. Hussain, M. F. Khan, J. Eom, J. Jung and Y. Seo, A progressive route for tailoring electrical transport in $\mathrm{MoS}_{2}$, Nano Res., 2016, 9(2), 380-391.

47 D. Shao, J. Gao, P. Chow, H. Sun, G. Xin, P. Sharma, J. Lian, N. A. Koratkar and S. Sawyer, Organic-inorganic heterointerfaces for ultrasensitive detection of ultraviolet light, Nano Lett., 2015, 15(6), 3787-3792.

48 R. B. Jacobs-Gedrim, M. Shanmugam, N. Jain, C. A. Durcan, M. T. Murphy, T. M. Murray, R. J. Matyi, R. L. Moore and B. Yu, Extraordinary photoresponse in two-dimensional In2Se3 nanosheets, ACS Nano, 2013, 8(1), 514-521.

49 F. Xia, T. Mueller, Y.-m. Lin, A. Valdes-Garcia and P. Avouris, Ultrafast graphene photodetector, Nat. Nanotechnol., 2009, 4(12), 839-843.

50 D.-S. Tsai, K.-K. Liu, D.-H. Lien, M.-L. Tsai, C.-F. Kang, C.-A. Lin, L.-J. Li and J.-H. He, Few-layer $\mathrm{MoS}_{2}$ with high broadband photogain and fast optical switching for use in harsh environments, ACS Nano, 2013, 7(5), 3905-3911.

51 P. Hu, Z. Wen, L. Wang, P. Tan and K. Xiao, Synthesis of fewlayer GaSe nanosheets for high performance photodetectors, ACS Nano, 2012, 6(7), 5988-5994.

52 P. Hu, J. Zhang, M. Yoon, X.-F. Qiao, X. Zhang, W. Feng, P. Tan, W. Zheng, J. Liu and X. Wang, Highly sensitive phototransistors based on two-dimensional GaTe nanosheets with direct bandgap, Nano Res., 2014, 7(5), 694-703. 
53 K. Nagashio, T. Yamashita, T. Nishimura, K. Kita and A. Toriumi, Electrical transport properties of graphene on $\mathrm{SiO}_{2}$ with specific surface structures, J. Appl. Phys., 2011, 110(2), 024513.

54 D. J. Late, B. Liu, H. R. Matte, V. P. Dravid and C. Rao, Hysteresis in single-layer $\mathrm{MoS}_{2}$ field effect transistors, ACS Nano, 2012, 6(6), 5635-5641.

55 Y.-H. Chang, W. Zhang, Y. Zhu, Y. Han, J. Pu, J.-K. Chang, W.-T. Hsu, J.-K. Huang, C.-L. Hsu and M.-H. Chiu, Monolayer MoSe2 grown by chemical vapor deposition for fast photodetection, ACS Nano, 2014, 8(8), 8582-8590.

56 C. Chen, H. Qiao, S. Lin, C. M. Luk, Y. Liu, Z. Xu, J. Song, Y. Xue, D. Li and J. Yuan, Highly responsive $\mathrm{MoS}_{2}$ photodetectors enhanced by graphene quantum dots, Sci. rep., 2015, 5, 11830.

57 C. Soci, A. Zhang, B. Xiang, S. A. Dayeh, D. Aplin, J. Park, X. Bao, Y.-H. Lo and D. Wang, ZnO nanowire UV photodetectors with high internal gain, Nano Lett., 2007, 7(4), 1003-1009.
58 L. Chen, F. Xue, X. Li, X. Huang, L. Wang, J. Kou and Z. L. Wang, Strain-gated field effect transistor of a $\mathrm{MoS}_{2}$ ZnO 2D-1D hybrid structure, ACS Nano, 2015, 10(1), 15461551.

59 R. Schlaf, O. Lang, C. Pettenkofer and W. Jaegermann, Band lineup of layered semiconductor heterointerfaces prepared by van der Waals epitaxy: charge transfer correction term for the electron affinity rule, J. Appl. Phys., 1999, 85(5), 2732-2753.

60 R. K. Chowdhury, R. Maiti, A. Ghorai, A. Midya and S. K. Ray, Novel silicon compatible p-WS 2 2D/3D heterojunction devices exhibiting broadband photoresponse and superior detectivity, Nanoscale, 2016, 8(27), 13429-13436.

61 V. A. Fonoberov, K. A. Alim, A. A. Balandin, F. Xiu and J. Liu, Photoluminescence investigation of the carrier recombination processes in $\mathrm{ZnO}$ quantum dots and nanocrystals, Phys. Rev. B: Condens. Matter Mater. Phys., 2006, 73(16), 165317. 